\title{
DEVELOPMENT OF THE PARTICULAR VeSSEl MATHEMATiCAL MODELS
}

\author{
Vadim Kramar
}
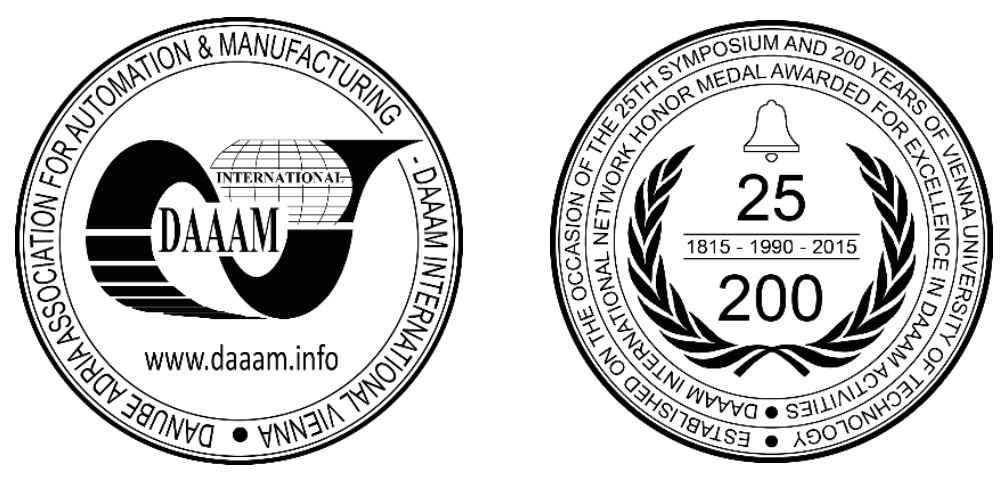

This Publication has to be referred as: Kramar, V[adim] (2016). Development of the Particular Vessel Mathematical Models, Proceedings of the 27th DAAAM International Symposium, pp.0034-0039, B. Katalinic (Ed.), Published by DAAAM International, ISBN 978-3-902734-08-2, ISSN 1726-9679, Vienna, Austria

DOI: $10.2507 / 27$ th.daaam.proceedings.005

\begin{abstract}
The technique of constructing a particular mathematical model of a given point vessel automatic deduction, taking into account the mutual influence ofmovement different type, as well as the impact of external factors, such as current, regular wave. The equations describe the motion of the vessel allows for different modes of operations performed. The particular vessel mathematical model was received that takes into account the operations performed on it at the time of its dynamic positioning.
\end{abstract}

Keywords: mathematical model; vessel;external factors;dynamic positioning control.

\section{Introduction}

Ships of various design and deadweight are used in today's worldwide practice for marine drilling aimed at probing or extracting minerals. There are automatic keeping systems over a drilling site in some of them. Some problems, such as, principles of automatic keeping systems, mathematical description of a drilling vessel as a control object of the automatic deduction contained require consideration in respect of theory as well as applying to technical implementation.

Development of ships mathematical models, taking into account the specificity of vessel design, equipment, including drilling, if we consider drilling vessel and process is an integral part of building a system of automatic deduction of a given point. When constructing mathematical models need to be sure to obtain the calculated ratios for the calculation of external forces and moments acting on the ship when it is spatial displacement, taking into account wind, currents, sea stat.

Contained in the various literature, for example [1-5], the output of a mathematical model of the vessel often overlapping and contains no presuppositions, which makes it difficult to carry out analytical studies and the formation of the algorithm for generating navigate a vessel when holding at a given point. Also, the information about the mathematical description of the vessel as a control object of the automatic deduction do not contain analytical relation vessels hydroaerodynamics characteristics and do not reveal the principles of such systems. Also, it is necessary to study the dynamics of the spatial movement of the ship hold. Obviously, at a specified vessel operation vessel may have different from each other dynamic models describing various process periods of operation. In this article, we will get particular mathematical models of the ship as well as the mathematical model of the vessel based on the current. 
Obtained in this paper a mathematical model of the vessel, taking into account such external influence as a trend, which is one of the major external factors hindering [5-7] the ship is at a given point during a given time allowed to become the basis of the algorithm for generating dynamic positioning control.

\section{Mathematical model of a vessel}

In [8] we obtain a generalized model ofthe interrelatedmovementsof the vessel on a regularwaves thatfor the studyof hisspeakersneed to be supplementedby equationsfor forcedcomponentsof vertical andhorizontaltransverse motion ofthe shipon a regular waves.In [8] we consideration the systems of coordinate, which characterize spatial position of the vessel and which is analog as [9].

Where all coefficients in (1) are obtain of the following arguments [8].

$$
\begin{aligned}
& \left(m+\lambda_{11}\right) \dot{v}_{x}+\dot{\lambda}_{11} v_{x}+m z_{c} \ddot{\psi}+\left[m \dot{z}_{c}+\left(m+\lambda_{33}\right) v_{z}+\mu_{33} \varsigma_{b}\right] \dot{\psi}- \\
& -\left[\left(m+\lambda_{22}\right) v_{y}+\mu_{22} \eta_{b} \cos \varphi\right] \dot{\varphi}=F_{x}, \\
& \left(m+\lambda_{22}\right) \dot{\mathrm{v}}_{y}+\dot{\lambda}_{22} \mathrm{v}_{y}-\left(m z_{c}-\lambda_{24}\right) \ddot{\theta}+\left[\mu_{22} \varsigma_{b}+\mu_{24}-m \dot{z}_{c}+\dot{\lambda}_{24}-\left(m+\lambda_{33}\right) \mathrm{v}_{z}-\mu_{33} \varsigma_{b}\right] \dot{\theta}+ \\
& +\left(\dot{\mu}_{22} \varsigma_{b}+\mu_{22} \dot{\zeta}_{b}+\dot{\mu}_{24}\right) \theta+\lambda_{26} \ddot{\varphi}+\left[\dot{\lambda}_{26}-\mu_{22} \eta_{b} \sin \varphi+\left(m+\lambda_{11}\right) v_{x}\right] \dot{\varphi}+\left(\dot{\mu}_{22} \eta_{b}+\mu_{22} \dot{\eta}_{b}\right) \cos \varphi=F_{y} \text {, } \\
& \left(m+\lambda_{33}\right) \dot{v}_{z}+\dot{\lambda}_{33} v_{z}+\lambda_{35} \ddot{\psi}+\left[\dot{\lambda}_{35}+\mu_{33} \eta_{b} \sin \varphi+\mu_{35}-\left(m+\lambda_{11}\right) v_{x}\right] \dot{\psi}+ \\
& +\left(\dot{\mu}_{33} \eta_{b} \sin \varphi+\mu_{33} \dot{\eta}_{b} \sin \varphi+\dot{\mu}_{35}\right) \psi+\left[\left(m+\lambda_{22}\right) v_{y}+\left(\mu_{22}-\mu_{33}\right) \eta_{b} \cos \varphi\right] \dot{\theta}- \\
& -\left(\dot{\mu}_{33} \eta_{b} \cos \varphi+\mu_{33} \dot{\eta}_{b} \cos \varphi\right) \theta+\mu_{35} \dot{\zeta}_{b}+\dot{\mu}_{35} \varsigma_{b}=F_{z}, \\
& \left(J_{x}+\lambda_{44}\right) \ddot{\theta}+\left[J_{x}+\dot{\lambda}_{44}+\mu_{24} \varsigma_{b}+\mu_{44}+\left(m z_{c}-\lambda_{24}\right) v_{z}-\dot{m} z_{c}^{2}\right] \dot{\theta}+ \\
& +\left[\dot{\mu}_{24} \varsigma_{b}+\mu_{24} \dot{\zeta}_{b}+\dot{\mu}_{44}-\mu_{33} \eta_{b} \cos \varphi v_{y}-\left(\mu_{24}+\mu_{22} \varsigma_{b}\right) v_{z}\right] \theta-\left(m z_{c}-\lambda_{24}\right) \dot{v}_{y}- \\
& -\left[m \dot{z}_{c}-\lambda_{24}-\left(\mu_{33} \eta_{b} \sin \varphi+\mu_{35}\right) \psi-\left(\lambda_{33}-\lambda_{22}\right) v_{z}-\left(\lambda_{35}+\lambda_{26}\right) \dot{\psi}-\mu_{33} \varsigma_{b}\right] v_{y}- \\
& -\left[\mu_{24} \eta_{b} \sin \varphi+\left(\lambda_{35}+\lambda_{26}\right) v_{z}+\mu_{35 \varsigma_{b}}+m z_{c} v_{x}\right] \dot{\varphi}+\left(\mu_{24} \dot{\eta}_{b}+\dot{\mu}_{24} \eta_{b}-\mu_{22} \eta_{b} v_{z}\right) \cos \varphi=M_{x} \text {, } \\
& \left(J_{y}+\lambda_{55}\right) \ddot{\psi}+\left(\dot{J}_{y}+\dot{\lambda}_{55}+\mu_{55}+\mu_{35} \eta_{b} \sin \varphi+m z_{c} v_{z}-\lambda_{35} v_{x}-\dot{m} z_{c}^{2}\right) \dot{\psi}+ \\
& +\left(\dot{\mu}_{55}+\dot{\mu}_{35} \eta_{b} \sin \varphi+\mu_{35} \dot{\eta}_{b} \sin \varphi-\mu_{33} \eta_{b} \sin \varphi v_{x}-\mu_{35} v_{x}\right) \psi+m z_{c} \ddot{v}_{x}+\left[m \dot{z}_{c}+\right. \\
& \left.+\left(\lambda_{11}-\lambda_{33}\right) v_{z}+\mu_{33}\left(\eta_{b} \cos \varphi \cdot \theta-\varsigma_{b}\right)\right] v_{x}+\lambda_{35} \dot{v}_{z}+\dot{\lambda}_{35} v_{z}-\left[\mu_{35} \eta_{b} \cos \varphi+\lambda_{26} v_{y}+\right. \\
& \left.+\mu_{26} \eta_{b} \cos \varphi\right) \dot{\theta}-\left(\dot{\mu}_{35} \eta_{b} \cos \varphi+\mu_{35} \dot{\eta}_{b} \cos \varphi\right) \theta+\left[\mu_{24} \eta_{b} \cos \varphi-\left(m z_{c}-\lambda_{24}\right) v_{y}\right] \dot{\varphi}+ \\
& +\mu_{35} \dot{\varsigma}_{b}+\dot{\mu}_{35} \varsigma_{b}=M_{y}, \\
& \left(J_{z}+\lambda_{66}\right) \ddot{\varphi}+\left(\dot{J}_{z}+\dot{\lambda}_{66}-\mu_{26} \eta_{b} \sin \varphi+\lambda_{26} v_{x}\right) \dot{\varphi}+\left(\dot{\mu}_{26} \eta_{b}+\mu_{26} \dot{\eta}_{b}-\mu_{24} \eta_{b} \dot{\psi}+\right. \\
& \left.+\mu_{22} \eta_{b} v_{x}\right) \cos \varphi+\lambda_{26} \dot{v}_{y}+\left[\dot{\lambda}_{26}-\lambda_{24} \dot{\psi}+\left(\lambda_{22}-\lambda_{11}\right) v_{x}\right] v_{y}+\left(\mu_{26} \varsigma_{b}+\dot{\lambda}_{35} v_{z}+\right. \\
& \left.+\mu_{35} \varsigma_{b}+\lambda_{24} v_{x}\right) \dot{\theta}+\left[\dot{\mu}_{26} \varsigma_{b}+\mu_{26} \dot{\varsigma}_{b}+\left(\mu_{22} \varsigma_{b}+\mu_{24}\right) v_{x}\right] \theta=M_{z}
\end{aligned}
$$

The movement of the vessel may be represented as consisting oftranslational motion with the starting point $G$ of the coordinate system, closely related to the vessel, and rotation around the point $G$. Vessel movement equations comprisemovement equations of the point $G$ dynamics, which coincides with the vessel gravity center at the moment, equations of rotation movement towards the point $G$ and equations ofthe gravity center dislocation in the course of the drilling operations.

In [8] we examine the movement of the vessel on the free infinite liquid surface and disregard liquid elasticity as a system "body+liquid in the vacuum" as it is carried out in $[9,10]$.

With the help of relations for $\vec{M}^{*}$ - the motion quantity instant vector of the particles and $\vec{F}^{*}$ - the motion quantity vectorof the particles, thrown off the body surface within a unit of time;

$$
\vec{M}^{*}=\sum_{v=1}^{n} \vec{r}_{v} \times \frac{d m_{v}}{d t} \vec{u}_{v}, \quad \vec{F}^{*}=\sum_{v=1}^{n} \frac{d m_{v}}{d t} \vec{u}_{v},
$$

where $\vec{u}_{v}$ is the absolute rate of the particles thrown offby a point having the mass $m_{v}, \vec{r}_{v}$ is the position vector of the point $m_{v}$ with respect to the static coordinate system, the plane of whichcoincides with theundisturbed freesurface of the liquid, and one axis is directed downward along the bore hole.

In problems about balancing on the water surface the following congruence may occur 


$$
\lambda_{j k}-\frac{i}{\sigma_{k}} \mu_{j k}=-\rho \iint_{\Omega} \varphi_{j} \frac{\partial \varphi_{k}}{\partial n} d \Omega \quad(j, k=1,2, \ldots, 6)
$$

where $\sigma_{k}$ is pitching frequency; $\lambda_{j k}$ and $\mu_{j k}$ are hydrodynamic coefficients which determine inertia and damping forces of wave nature, the functions $\varphi_{i}(x, y)$ depend on oscillation frequency and rate of sailing

On denoting $v_{g x}=v_{1}, v_{g y}=v_{2}, v_{g z}=v_{3}, \omega_{x}=v_{4}, \omega_{y}=v_{5}, \omega_{z}=v_{6}$, we obtain

$$
\Phi=\sum_{i=1}^{6} v_{i} \varphi_{i}
$$

where $\Phi$ is the potential offended progressive waves velocities.

To go further we shall discuss a series of assumptions. Let us assume that: 1) The angles $\theta$ and $\psi$ are small, therefore $\sin \theta=\theta, \sin \psi=\psi, \cos \theta=\cos \psi=1 ; 2)$ movement through the points $\psi$ and $\theta$ are forced and cyclic with oscillation frequency $\sigma_{k}$, depending on the regular waves frequency $\sigma$ and the vessel velocity $v: \sigma_{k}=\sigma-v k \cos \chi$, where $k$ is the module of sea wave shape, $\chi$ - the angle between the direction of the vessel and that of wave advance; 3 ) movementalong the verticaland transversehorizontalcoordinates in thefixed coordinate system consists ofthe cyclic component( frequency $\sigma_{k}$ ) and the noncyclic one; 4) we disregard damping fluid properties towards the axis $O \xi ; 5$ ) the elements containing products of the values $\psi, \dot{\psi}, \theta, \dot{\theta}, \dot{\varphi}, \ddot{\varphi}$ are small and can be disregarded.

In accordance with the assumptions accepted we can formulate:

Having small values $\theta, \psi, \dot{\varphi}$ for angular and linear rates in the related standard of rest we obtain

$$
\begin{aligned}
\omega_{x}= & \dot{\theta} ; \omega_{y}=\dot{\psi} ; \omega_{z}=\dot{\varphi} ; \\
v_{x}= & a_{1} \dot{\xi}_{g}+a_{2} \dot{\eta}_{g}+a_{3} \dot{\zeta}_{g}=\dot{\xi}_{g} \cos \varphi+\dot{\eta}_{c} \sin \varphi+i \sigma_{k}\left(\eta_{b} \sin \varphi+\sigma_{k}^{-2} \dot{\psi}_{g}\right) ; \\
v_{y}= & b_{1} \dot{\xi}_{g}+b_{2} \dot{\eta}_{g}+b_{3} \dot{\zeta}_{g}=-\dot{\xi}_{g} \sin \varphi+\dot{\eta}_{c} \cos \varphi+i \sigma_{k}\left(\eta_{b} \cos \varphi+\varsigma_{b} \theta\right)+\theta \dot{\zeta}_{c} ; \\
v_{z}= & c_{1} \dot{\xi}_{g}+c_{2} \dot{\eta}_{g}+c_{3} \dot{\zeta}_{g}=(\psi \cos \varphi+\theta \sin \varphi) \dot{\xi}_{g}+(\psi \sin \varphi-\theta \cos \varphi) \dot{\eta}_{g}+\dot{\zeta}_{c}+ \\
& \quad+i \sigma_{k}\left[\eta_{b}(\psi \sin \varphi-\theta \cos \varphi)+\varsigma_{b}\right] .
\end{aligned}
$$

Writing at small values $\theta, \psi, \dot{\varphi}$ the expressions for the angular and linear velocity related coordinate system, substituting them into the expression for $R_{x}, R_{y}, R_{z}, N_{x}, N_{y}, N_{z}$ and taking into account only thereall partsget

$$
\begin{aligned}
& R_{x}=\left(m+\lambda_{11}\right) v_{x}+m z_{c} \dot{\psi} ; \\
& R_{y}=\left(m+\lambda_{22}\right) v_{y}-\left(m z_{c}-\lambda_{24}\right) \dot{\theta}+\left(\mu_{22} \zeta_{b}+\mu_{24}\right) \theta+\lambda_{26} \dot{\varphi}+\mu_{22} \eta_{b} \cos \varphi ; \\
& R_{z}=\left(m+\lambda_{33}\right) v_{z}+\lambda_{35} \dot{\psi}+\left(\mu_{33} \eta_{b} \sin \varphi+\mu_{35}\right) \psi-\mu_{33} \eta_{b} \theta \cos \varphi+\mu_{33} \zeta_{b} ; \\
& N_{x}=\left(J_{x}+\lambda_{44}\right) \dot{\theta}-\left(m z_{c}-\lambda_{24}\right) v_{y}+\mu_{24}\left(\eta_{b} \cos \varphi+\theta \zeta_{b}\right)+\mu_{44} \theta \\
& N_{y}=\left(J_{y}+\lambda_{55}\right) \dot{\psi}+\left(\mu_{55}+\mu_{35} \eta_{b} \sin \varphi\right) \psi+m z_{c} v_{x}+\lambda_{35} v_{z}-\mu_{35} \eta_{b} \theta \cos \varphi+\mu_{35} \xi_{b} \\
& N_{z}=\left(J_{z}+\lambda_{66}\right) \dot{\varphi}+\lambda_{26} v_{y}+\mu_{26}\left(\eta_{b} \cos \varphi+\zeta_{b} \theta\right) .
\end{aligned}
$$

Substituting (6) inequation of the equation for projections of motion quantity andmotion quantity instants of the particles, we obtain [8]a generalized model (1) ofthe interrelatedmovementsof the vesselon a regularwaves thatfor the studyof hisspeakersneed to be supplementedby equationsfor forcedcomponentsof vertical andhorizontaltransverse motion ofthe shipon a regular waves.

The system of equations(1) should be supplemented by equations for forced components $\varsigma_{b}, \eta_{b}$ of vertical and horizontal transverse motion of the ship on a regular excitement

$$
\dot{\eta}_{b}=-\sigma_{k} \eta_{0}(r, \sigma, \chi) \sin \sigma_{k} t, \quad \dot{\zeta}_{b}=-\psi v_{x}+\theta v_{y}+v_{z}-\dot{z}_{c} .
$$

Here $\chi$ - the angle between the direction of wave propagation and the longitudinal axis of the vessel. To determine the amplitude $\eta_{0}(r, \sigma, \chi)$ as a function of the parameters of the sea waves, can be applied dependence [3] 
$\eta_{0}(r, \sigma, \chi)=\aleph_{n} \eta_{1}$

where $\aleph_{n}$-reduction factor, which is calculated based on the geometric dimensions of the ship and sea waves parameters is performed according to the method proposed in [5]; $\eta_{1}$ - the amplitude of the wave-particle oscillations in the direction of wave propagation.

The system of integrated equations of the drilling vessel changes, obtained in [8], is not an analogue of a real vessel, as the latter, depending on the type of operations performed, may have one of the four dynamic analogues corresponding to the stages(initial, preliminary, drilling, ultimate) of drilling activity.

So, on assuming the dimension $m, \lambda_{i j}, \mu_{i j}, J_{x}, J_{y}, J_{z}, z_{c}$ from (1)as constant and regarding the equation

$$
\dot{\xi}_{g}=a_{1} v_{x}+b_{1} v_{y}+c_{1} v_{z}, \quad \dot{\eta}_{g}=a_{2} v_{x}+b_{2} v_{y}+c_{2} v_{z}, \quad \dot{\zeta}_{g}=a_{3} v_{x}+b_{3} v_{y}+c_{3} v_{z},
$$

we obtain the equation of the vessel changes at the initial stage.

To obtain the equations reflecting vessel movement at the preliminary period, if we consider drilling vessel [8] we write down:

$$
\begin{array}{ll}
\psi=\psi_{0} e^{j \sigma_{k} t}, & \theta=\theta_{0} e^{j \sigma_{k} t}, \\
\zeta_{g}=\zeta_{0} e^{j \sigma_{k} t}, & \eta_{g}=\eta_{0} e^{j \sigma_{k} t}
\end{array}
$$

On assuming the dimensions $m, \lambda_{i j}, \mu_{i j}(i, j=1,2, \ldots, 6) J_{x}, J_{y}, J_{z}, z_{c}$ as constant, on carrying out calculations similar to (8)- (10) we obtain equations of the preliminary period.

$$
\begin{aligned}
& \left(m+\lambda_{11}\right) \dot{v}_{x}+m z_{c} \ddot{\psi}+\left[\left(m+\lambda_{33}\right) v_{z}+\mu_{33} \varsigma_{b}\right] \dot{\psi}- \\
& -\left[\left(m+\lambda_{22}\right) v_{y}+\mu_{22} \eta_{b} \cos \varphi\right] \dot{\varphi}=F_{x}, \\
& \left(m+\lambda_{22}\right) \dot{v}_{y}+\left(\lambda_{24}-m z_{c}-\left(\mu_{22} / \sigma_{k}^{2}\right) v_{z}\right) \ddot{\theta}+\left[\mu_{22} \varsigma_{g}-\left(\mu_{22} / \sigma_{k}^{2}\right) \dot{v}_{z}+\right. \\
& \left.+\mu_{24}-\left(m+\lambda_{33}\right) v_{z}-\mu_{33} \varsigma_{b}\right] \dot{\theta}+\mu_{22} v_{z} \theta+\lambda_{26} \ddot{\varphi}+\lambda_{26} \ddot{\varphi}+ \\
& +\left[\left(m+\lambda_{11}\right) v_{x}-\mu_{22} \eta_{g} \sin \varphi\right] \dot{\varphi}+\mu_{22} \dot{\eta}_{b} \cos \varphi=F_{y}, \\
& \left(m+\lambda_{33}\right) \dot{v}_{z}+\mu_{33} v_{z}+\left(\lambda_{35}-\left(\mu_{33} / \sigma_{k}^{2}\right) v_{x}\right) \ddot{\psi}+\left[\mu_{35}-\left(\mu_{33} / \sigma_{k}^{2}\right) \dot{v}_{x}-\left(m+\lambda_{11}\right) v_{x}\right] \dot{\psi}+ \\
& +\left(\mu_{33} / \sigma_{k}^{2}\right) v_{y} \ddot{\theta}+\left[\left(\mu_{33} / \sigma_{k}^{2}\right) \dot{v}_{y}+\left(m+\lambda_{22}\right) v_{y}+\mu_{22} \eta_{g} \cos \varphi\right] \dot{\theta}=F_{z}, \\
& \left(J_{x}+\lambda_{44}-\left(\mu_{24} / \sigma_{k}^{2}\right) v_{z}\right) \ddot{\theta}+\left[\mu_{44}+\mu_{24} \zeta_{g}-\left(\mu_{24} / \sigma_{k}^{2}\right) \dot{v}_{z}+\left(m z_{c}-\lambda_{24}\right) v_{z}+\left(\mu_{33} / \sigma_{k}^{2}\right) v_{y}^{2}\right] \dot{\theta}- \\
& -\mu_{22} \varsigma_{g} v_{z} \theta+\left[\left(\lambda_{26}+\lambda_{35}-\left(\mu_{33} / \sigma_{k}^{2}\right) v_{x}\right) v_{y}+\mu_{26} \eta_{g} \cos \varphi\right] \dot{\psi}+\mu_{35} v_{y} \psi- \\
& -\left[\mu_{24} \eta_{g} \sin \varphi+m z_{c} v_{x}+\left(\lambda_{35}+\lambda_{26}\right) v_{z}+\mu_{35} \varsigma_{g}\right] \dot{\varphi}+\left(\lambda_{24}-m z_{c}\right) \dot{v}_{y}+\mu_{33} \varsigma_{g} v_{y}+ \\
& +\left[\left(\lambda_{33}-\lambda_{22}\right) v_{y}-\mu_{22} \eta_{g} \cos \varphi\right] v_{z}+\mu_{24} \dot{\eta}_{g} \cos \varphi=M_{x} \\
& \left(J_{y}+\lambda_{55}+\left(\mu_{35} / \sigma_{k}^{2}\right) v_{x}\right) \ddot{\psi}+\left[m_{z} v_{z}-\left(\lambda_{35}-\left(\mu_{35} / \sigma_{k}^{2}\right) v_{x}\right) v_{x}+\mu_{55}-\left(\mu_{35} / \sigma_{k}^{2}\right) \dot{v}_{x}\right] \dot{\psi}- \\
& -\mu_{35} v_{x} \psi+\left(\mu_{35} / \sigma_{k}^{2}\right) v_{y} \ddot{\theta}+\left[\left(\mu_{35} / \sigma_{k}^{2}\right) \dot{v}_{y}-\lambda_{26} v_{y}-\mu_{26} \eta_{g} \cos \varphi-\left(\mu_{35} / \sigma_{k}^{2}\right) v_{x} v_{y}\right] \dot{\theta}+ \\
& +\left[\left(-m z_{c}+\lambda_{24}\right) v_{y}+\mu_{24} \eta_{g} \cos \varphi\right] \dot{\varphi}+m z_{c} \dot{v}_{x}+\left[\left(\lambda_{11}-\lambda_{33}\right) v_{z}-\mu_{33} \zeta_{g}\right] v_{x}+\lambda_{35} \dot{v}_{z}+\mu_{35} v_{z}=M_{y}, \\
& \left(J_{z}+\lambda_{66}\right) \ddot{\varphi}+\left(\lambda_{26} v_{x}-\mu_{26} \eta_{g} \sin \varphi\right) \dot{\varphi}+\left(\mu_{26} / \sigma_{k}^{2}\right) v_{z} \ddot{\theta}+\left[\mu_{26} \eta_{g}-\left(\mu_{26} / \sigma_{k}^{2}\right) \dot{v}_{z}+\lambda_{35} v_{z}+\mu_{24} \eta_{b} \dot{\psi}+\right. \\
& \left.+\mu_{35} \varsigma_{g}-\left(\mu_{26} / \sigma_{k}^{2}\right) v_{z} v_{x}+\lambda_{24} v_{x}\right) \dot{\theta}+\left[\mu_{26} v_{z}+\left(\mu_{22} \varsigma_{g}+\mu_{24}\right) v_{x}\right] \theta-\left(\lambda_{24} v_{y}+\mu_{24} \eta_{g} \cos \varphi\right) \dot{\psi}+ \\
& +\lambda_{26} \dot{v}_{y}+\mu_{26} \eta_{g} \cos \varphi+\left[\left(\lambda_{22}-\lambda_{11}\right) v_{y}+\mu_{22} \eta_{g} \cos \varphi\right] v_{x}=M_{z} \text {. } \\
& \dot{\xi}_{g}=v_{x} \cos \varphi-v_{y} \sin \varphi+(\psi \cos \varphi+\theta \sin \varphi) v_{z} \text {, } \\
& \dot{\eta}_{g}=v_{x} \sin \varphi+v_{y} \cos \varphi+(\psi \sin \varphi-\theta \cos \varphi) v_{z}, \\
& \dot{\zeta}_{g}=-\psi v_{x}+\theta v_{y}+v_{z} \text {. }
\end{aligned}
$$


Now, let us assume that current is possible only in the horizontal plane and it is characterized by velocity $v_{T}$ and direction (the angle $\alpha_{T}$ between the velocity vector $\bar{v}_{T}$ and the axis $O \xi$ ) that is its components:

$$
\dot{\xi}_{T}=v_{T} \cos \alpha_{T}, \dot{\eta}_{T}=v_{T} \sin \alpha_{T}, \dot{\zeta}_{T}=\zeta_{T}=0
$$

In the projections upon dynamic axes of reference Gxyz we obtain:

$$
\begin{aligned}
& v_{x T}=a_{1} \dot{\xi}_{T}+a_{2} \dot{\eta}_{T}+a_{3} \dot{\zeta}_{T}=v_{T} \cos \left(\alpha_{T}-\varphi\right), \\
& v_{y T}=b_{1} \dot{\xi}_{T}+b_{2} \dot{\eta}_{T}+b_{3} \dot{\zeta}_{T}=v_{T} \sin \left(\alpha_{T}-\varphi\right), \\
& v_{z T}=c_{1} \dot{\xi}_{T}+c_{2} \dot{\eta}_{T}+c_{3} \dot{\zeta}_{T}=-v_{T} \theta \sin \left(\alpha_{T}-\varphi\right)+v_{T} \psi \cos \left(\alpha_{T}-\varphi\right) .
\end{aligned}
$$

Here $a_{i}, b_{i}, c_{i},(i=1,2,3)$ are factors determined through the above-mentioned anglesby the equation

$$
\begin{aligned}
& a_{1}=\cos \varphi \cos \psi, a_{2}=\sin \varphi \cos \psi, a_{3}=\sin \psi, \\
& b_{1}=\sin \theta \cos \varphi \sin \psi-\cos \theta \sin \varphi, b_{2}=\cos \theta \cos \varphi+\sin \theta \sin \varphi \sin \psi, b_{3}=\sin \theta \cos \varphi, \\
& c_{1}=\cos \theta \cos \varphi \sin \psi+\sin \theta \sin \varphi, c_{2}=\cos \theta \sin \varphi \sin \psi-\sin \theta \cos \varphi, c_{3}=\cos \theta \cos \psi
\end{aligned}
$$

The resultant velocity of the vessel will be composed of vessel velocity $\vec{v}$ regardless of the current and current velocity $\vec{v}_{T}$. The components of the resultant vessel velocity in the dynamic standard of rest are determined through the equation:

$$
\begin{aligned}
& v_{x 1}=v_{x}+v_{x T}=v_{x}+v_{T} \cos \left(\alpha_{T}-\varphi\right), \\
& v_{y 1}=v_{y}+v_{y T}=v_{y}+v_{T} \sin \left(\alpha_{T}-\varphi\right), \\
& v_{z 1}=v_{z}+v_{z T}=v_{z}-v_{T} \sin \left(\alpha_{T}-\varphi\right)+v_{T} \psi \cos \left(\alpha_{T}-\varphi\right) .
\end{aligned}
$$

On plugging the formulations (15) into the equation (9), we obtainequations ofvessel gravity center displacement in the static standard of rest $O \xi \eta \zeta$ regarding the current:

$$
\begin{aligned}
& \dot{\xi}_{g}=v_{x} \cos \varphi-v_{y} \sin \varphi+(\psi \cos \varphi+\theta \sin \varphi) v_{z}+v_{T} \cos \alpha_{T} \\
& \dot{\eta}_{g}=v_{x} \sin \varphi+v_{y} \cos \varphi+(\psi \sin \varphi-\theta \cos \varphi) v_{z}+v_{T} \sin \alpha_{T} \\
& \dot{\zeta}_{g}=-\psi v_{x}+\theta v_{y}+v_{z} .
\end{aligned}
$$

\section{Conclusion}

In this article, we received a particular vessel mathematical model that takes into account the operations performed on it at the time of its dynamic positioning. For example, if we consider drilling vessel, depending on the type of work undertaken vessel can have one of four dynamic models corresponding periods (initial, preparatory drilling, end) drilling. Also, when constructing a mathematical model of vessel movement is obviously necessary to consider external factors. This article discusses the current account and the regular excitement in the construction of a mathematical model. To build a complete model of the need to integrate marine equipment used during operations on the parameters of the equations of dynamics of the vessel.

\section{References}

[1] Fossen, Thor I.,Guidance and Control of Ocean Marine Vehicles. John Wiley and Sons Ltd.New York, 1994.

[2] Lewis, E.V., Principles of Naval Architecture vol III: Motions in Waves and Controllability. Society of NavalArchitecture and Marine Engineers, New York,1988.

[3] Jensen, Andreas G. Fluid dynamic derivatives: marine and wind engineering approaches. Journal of Wind Eng.and Industrial Aerodynamics 69 (1997) 777-793.

[4] Abkowitz, M.A. Lectures on ship hydrodynamics- steering and manoeuvrability. Technical Report Rep.No.Hy5.Hydro ogAerodynamiskLaboratorium, Lyngby, Denmark, 1975.

[5] Ruoshi Cha, Decheng Wan, Numerical Investigation of Motion Response of Two Model Ships in Regular Waves. Procedia Engineering, Volume 116, 2015, pp. 20-31. DOI:10.1016/j.proeng.2015.08.260 
[6] Ivanova O., Dushko V., Rodkina A. Experimental Researches Automation of Spatial Oscillations of the Floating Ocean Engineering Systems in the Wave Basin. Proceedings of the 26th DAAAM International Symposium, pp.1059-1067,B. Katalinic (Ed.), Published by DAAAM International, ISBN 978-3-902734-07-5, ISSN 1726-9679, Vienna, AustriaDOI:10.2507/26th.daaam.proceedings.149.

[7] Nesin Danilo Y., Dushko Veronica R. Numerical model of the large carrying capacity crane ship with the fullyrevolving topside. Procedia Engineering (25th DAAAM International Symposium on Intelligent Manufacturing and Automation, DAAAM 2014), Volume 100, 2015, Pages 1082-1091.

[8] Kramar V. Investigation of Changes and Development of Mathematical Model for a Drilling Vessel. Proceedings of the 26th DAAAM International Symposium, pp.0049-0055,B. Katalinic (Ed.), Published by DAAAM $\begin{array}{lllll}\text { International, } \quad \text { ISBN } & 978-3-902734-07-5, & \text { ISSN } & \text { 1726-9679, }\end{array}$ AustriaDOI:10.2507/26th.daaam.proceedings.007

[9] T. Perez, A. J. Sørensen, and M. Blanke.Marine Vessel Models in Changing Operational Conditions - A Tutorial.In Proceedings of the 16th IFAC Symposium on System Identi_cation, Newcastle, Australia, 2006.

[10] Blagoveshensky S.N. Handbook of statics and dynamics of the ship. Shipbuilding Publishers, St. Petersburg,Russia,1976. 